\title{
ECG Signal Compression: A Transform Based Approach
}

\author{
Sulbha N. Thorat ${ }^{1}$, Prof. Supriya Rajankar ${ }^{2}$ \\ ${ }^{I}$ (E\&TC, SCOE/ Pune University, India) \\ ${ }^{2}(E \& T C, S C O E /$ Pune University, India)
}

\begin{abstract}
Over the years, a variety of other linear transforms have been developed which include discrete Fourier transform (DFT), discrete wavelet transform (DWT) and discrete cosine transform (DCT) and many more, each with its own advantages and disadvantages. Among these techniques, DWT has been proven to be very efficient for ECG signal coding. It is proposed to develop, a hybrid two-stage ECG signal compressor based on DCT and DWT. Proposed method is hybrid ECG compression technique based on wavelet transformation of the DCT coefficients of the signal. Interaction of DCT analysis with DWT transformations, signal thresholding and coding are a few of the many outstanding challenges in ECG compression. Proposed method will offer optimized CR and PRD, which would be suitable for most monitoring and diagnostic applications.
\end{abstract}

Keywords- DCT, DWT, Wavelet, Image Processing, Signal Compression.

\section{Introduction}

The central goal of electrocardiogram (ECG) [1] data compression techniques is to preserve the most useful diagnostic information while compressing a signal to an acceptable size. Lossless compression is the best choice as long as the compression ratio is acceptable, but it cannot usually offer a satisfactory compression ratio (CR). To obtain significant signal compression, lossy compression is preferable to a lossless compression. Lossless compression is nothing but exact reconstruction of the original signal and is based on the idea of breaking a file into a "smaller" form for transmission or storage and then putting it back together on the other end so it can be used again. Whereas lossy compression simply eliminate "unnecessary" bits of information, that's why for compressing an ECG signal lossy is preferred. In this case, compression is accomplished by applying an invertible orthogonal transform to the signal, and one tries to reduce the redundancy present in the new representation. Due to its decorrelation and energy compaction properties and to the existence of efficient algorithms to compute it, discrete cosine transform have been widely investigated for ECG signal compression \& DWT [5] \& [6] has been proven to be very efficient for ECG signal coding.

In this paper, a hybrid [4] two-stage ECG signal compression method based on DCT and DWT is proposed. Their combination removes the spectral redundancy by compressing the subordinate components more than the dominant components. The resulting transformed coefficients that represent the transformational signal are then threshold and compressed using a new coding technique for storage space saving.

\section{Hybrid Method}

Hybrid method is nothing but the combination of DCT and DWT methods. ECG samples are applied to DCT-IV which is a linear orthogonal lapped transform, based on the idea of time domain aliasing cancellation (TDAC). It is designed to be performed on consecutive blocks of a larger dataset, where subsequent blocks are overlapped so that the last half of one block coincides with the first half of the next block. This overlapping, in addition to the energy-compaction qualities of the DCT, makes DCT-IV especially attractive for signal compression applications. Thus, it helps to avoid artifacts stemming from the block boundaries. DCT-IV is critically sampled, which means that though it is 50\% overlapped, a sequence data after DCT-IV has the same number of coefficients as samples before the transform (after overlap-and-add). This means that a single block of IDCT data does not correspond to the original block on which the DCT-IV was performed. When subsequent blocks of inverse transformed data are added, the errors introduced by the transform cancel out TDAC.

DCT-IV is defined as:

$$
\begin{gathered}
\mathrm{X}_{\mathrm{c}}(\mathrm{k})=\sum_{\mathrm{n}=0}^{\mathrm{N}-1} x(\mathrm{n}) \cos \left[\left(\mathrm{n}+\frac{\mathrm{M}+1}{2}\right)\left(\mathrm{k}+\frac{1}{2}\right) \frac{\pi}{\mathrm{M}}\right] \\
\text { where, } \quad \mathrm{k}=0,1, \ldots \mathrm{M}-1
\end{gathered}
$$


Where, $x(n), n=0,1,2, \ldots, N$ - 1 is the sequence to be transformed, $N=2 M$ is the window length and $M$ is the number of transform coefficients. The computation burden can be reduced if the transform coefficients given by equation (2) are rewritten in the following recursive form:

$$
\mathrm{Xc}(\mathrm{k})=\mathrm{x}(0) \cos \left[(\mathrm{M}+1) \frac{\Theta_{\mathrm{k}}}{2}\right]+V_{1} \cos \left[(M+3) \frac{\theta_{k}}{2}\right]-\mathrm{V}_{2} \cos \left[(\mathrm{M}+1) \frac{\Theta_{\mathrm{k}}}{2}\right]
$$

where,

and

$$
\begin{array}{r}
\mathrm{V}_{\mathrm{m}}=x(\mathrm{~m})+2 \cos \theta_{\mathrm{k}} \mathrm{V}_{\mathrm{m}+1}-\mathrm{V}_{\mathrm{m}+2} \\
\mathrm{~m}=\mathrm{N}-1, \mathrm{~N}-2, \ldots .1,0,
\end{array}
$$

$$
\theta_{\mathrm{k}}=\left(\mathrm{k}+\frac{1}{2}\right) \frac{\pi}{\mathrm{M}}
$$

The DCT-IV computation algorithm of a data sequence $x(n)$ can be summarized by the following:

a) Partition the data sequence in $\mathrm{N}_{\mathrm{b}}$ consecutive blocks, each one with $\mathrm{N}=64$ samples.

b) Recursively generate the $V_{m}$ from the input sequence $x(n)$ according to equations (3) and (4).

c) Calculate the DCT-IV coefficients for each block by evaluating the $k$ th DCT-IV coefficient using equation (2) at the $N$ th step.

In the decompression stage, inverse DCT-IV, termed IDCT-IV, is adopted. Because there are different numbers of inputs and outputs, at first glance it might seem that DCT should not be invertible. However, perfect invertability is achieved by adding the overlapped IDCT-IV s of subsequent overlapping blocks, causing the errors to cancel and the original data to be retrieved. IDCT-IV transforms the $M$ real coefficients, $X_{C}(0)$, $X_{C}(1), \ldots, X_{C}(M-1)$, into $N=2 M$ real numbers, $x(0), x(1), \ldots, x(N-1)$, according to the Formula:

$$
\begin{gathered}
\mathrm{x}(\mathrm{n})=\sum_{\mathrm{k}=0}^{\mathrm{M}-1} \mathrm{X}_{\mathrm{c}}(\mathrm{k}) \cos \left[\left(\mathrm{n}+\frac{\mathrm{M}+1}{2}\right)\left(\mathrm{k}+\frac{1}{2}\right) \frac{\pi}{\mathrm{M}}\right] \\
\mathrm{n}=0,1, \ldots . \mathrm{N}-1
\end{gathered}
$$

Again, the computation burden of $x(n)$ can be reduced considerably if equation (5) is rewritten in the following recursive form:

$$
x(n)=X_{c}(0) \cos \left[\frac{\left[\theta_{n}\right]}{2}\right]+V_{1} \cos \left[\frac{3 \theta_{n}}{2}\right]-V_{2} \cos \left[\frac{\theta_{n}}{2}\right]
$$

Where,

$$
\begin{gathered}
\mathrm{V}_{\mathrm{m}}=\mathrm{X}_{\mathrm{c}}(\mathrm{m})+\cos \theta_{\mathrm{n}} \mathrm{V}_{\mathrm{m}+1}-\mathrm{V}_{\mathrm{m}+2} \\
\theta_{\mathrm{n}}=\left(n+\frac{M+1}{2}\right) \frac{\pi}{M}
\end{gathered}
$$

\subsection{Discrete Cosine Transform}

Generally, in transform-based ECG compression methods, an invertible orthogonal transformation is applied to the signal, and one tries to reduce the redundancy present in the new representation. Due to their decorrelation and energy compaction properties and to the existence of efficient algorithms to compute them, Cosine and Wavelet transforms have been widely investigated for data compression. The DCT, for example, has been used for ECG, image, video, and audio compression.

There are normally 4 general steps in a DCT-based compression of a data sequence $\mathrm{x}$,

a) Partition of $\mathrm{x}$ in $N_{\mathrm{b}}$ consecutive blocks $\mathrm{b}_{\mathrm{i}}, i=0,1 \ldots N b_{-} 1$, each one with $L \mathrm{~b}$ samples;

b) DCT computation for each block;

c) Quantization of the DCT coefficients;

d) Lossless encoding of the quantized DCT coefficients.

Increasing the block size increases the CR and the DCT computing time. Various results show, however, that increasing the block size above a certain point results in a very modest CR gain, while the 
processing time significantly increases. The type II DCT (DCT-II) is commonly used for data compression due to its greater capacity to concentrate the signal energy in few transform coefficients.

\subsection{Discrete Wavelet Transform}

Recently developed wavelet transforms have become an attractive and efficient tool in many applications especially in coding and compression of signals. This results from their multi-resolution and highenergy compaction properties. Wavelet transform can be viewed as a block transform with overlapping basis functions of variable lengths.

Using WT the time domain signal $x(t)$ can be expanded in terms of a weighted sum of a set of basis functions, in a way similar to the generalized Fourier series expansion. While for the classical Fourier series the basis functions are sine and cosine functions at different frequencies, in the case of wavelet series, the basis functions $\Psi_{j} k(t)$ are translations and dilations of a single fixed function $\Psi(t)$ called the mother wavelet.

$$
\Psi_{j k}(t)=2^{j / 2} \psi\left(2^{j} t-k\right), \quad j, k \in Z
$$

For certain choices of $\Psi(t)$, the corresponding set of $\Psi_{j k}(t)$ forms an orthonormal basis in $L 2(R)$. In this case,

$$
\mathrm{x}(t)=\sum_{j} \sum_{k} d_{j k} \psi_{j k}(t)
$$

Where the wavelet coefficients $d_{j k}$ are calculated using the relation

$$
d_{j k}=\int x(t) \Psi_{j k}(t) d t=2^{\frac{j}{2}} \int x(t) \psi\left(2^{j} t-k\right) d t
$$

In contrast to the sine and cosine, wavelets are local in frequency/scale via dilations and in time via translations. This localization offers an advantage, since fewer wavelet basis functions are usually needed to represent the signal $x(t)$ to a given level of approximation. This property is of great importance in the compression of the signal. For fast numerical computations the signal is sampled at discrete time instants $t_{t}=i / L$ where $L$ is the signal length.

\section{Quantization and Coding Algorithms}

A quantizers simply reduces the number of bits needed to store the transformed coefficients by reducing the precision of those values. A quantization scheme maps a large number of input values into a smaller set of output values. This implies that some information is lost during the quantization process. The original wavelet coefficients $c(n)$ cannot be recovered exactly after quantization.

\subsection{Energy Packing Efficiency}

In this section, the quantization strategy adopted is based on the energy packing efficiency (EPE). It guarantees the balance between the compression achievement and information loss. Here, quantization process is performed by selecting an appropriate threshold level $\lambda$ to control the compression ratio. Due to careful representation by combining the DCT and DWT, it is reasonable to assume that only a few coefficients contain information about the real signal while others appear as less important details. The goal is to extract these significant coefficients and to ignore others smaller than specified threshold level $\lambda$. The optimal value of $\lambda$ is determined such that the reconstructed signal is as close to the original one as possible. Usually the selection of optimal threshold level is not an easy task, because some of the coefficients that represent the actual signal details may be also killed, and as a result, signal distortion is the side-effect. As can be deduced from the above discussion, the approximation band is the smallest band in size and it includes high amplitude approximation coefficients. The wavelet coefficients other than these included in the approximation band, detail coefficients, have small magnitudes. Most of the energy is captured by these coefficients of the lowest resolution band. Here, threshold levels are defined according to the energy packing efficiencies of the signal for all sub bands. EPE for a set of coefficients in the $i_{\mathrm{th}}$ sub band is defined as the ratio of the energy captured by the sub band coefficients and the energy captured by the whole number of coefficients.

$$
\mathrm{EPE}_{\mathrm{i}}=\frac{\sum_{\mathrm{n}=1}^{\mathrm{L}_{\mathrm{i}}}(\mathrm{c}(\mathrm{n}))^{2}}{\sum_{\mathrm{n}=1}^{\mathrm{L}}(\mathrm{c}(\mathrm{n}))^{2}} \times 100
$$


Where $\mathrm{Li}$ and $\mathrm{L}$ are the number of coefficients in the $\mathrm{i}^{\text {th }}$ sub band and the whole number of coefficients respectively.

An encoder further compresses the quantized values losslessly to give better overall compression. The most commonly used encoders are the Huffman encoder and the arithmetic encoder, although for applications requiring fast execution, simple run-length encoding (RLE) has proven very effective.

\section{Compression and Distortion Measures}

All data compression algorithms seek to minimize data storage by eliminating redundancy where possible by increasing the compression ratio. The latter is defined as the ratio of the number of bits representing the original signal to the number of bits required to store the compressed signal. A high compression ratio is typically desired, but using this alone to compare data compression algorithms is not acceptable. Moreover, the bandwidth, sampling frequency, and precision of the original data very much affect the compression ratio. A data compression algorithm must also represent the data with acceptable fidelity. In ECG signal compression, the clinical acceptability of the reconstructed signal has to be determined through visual inspection by cardiologists. The error signal resulting from the difference between the reconstructed signal and the original one may also be measured numerically. A lossless data compression algorithm produces zero residual, and the reconstructed signal exactly replicates the original signal. However, clinically acceptable quality is neither guaranteed by a low nonzero residual nor ruled out by a high numerical residual. The criteria for testing compression algorithms performance include three components: compression measure, reconstruction error and computational complexity. The compression measure and the reconstruction error are usually dependent on each other and are used to create the rate-distortion function of the algorithm. The computational complexity component is part of the practical implementation consideration.

\subsection{Distortion Measurement}

One of the most difficult problems in ECG compression and reconstruction is defining the error criterion that measures the ability of the reconstructed signal to preserve the relevant information. As yet, there is no mathematical structure to this criterion, and all accepted error measures are still variations of the mean square error or absolute error, which are easy to compute mathematically, but are not always diagnostically relevant. In technical literature, the distortion resulting from the ECG processing is frequently measured by the percentage rms difference (PRD). It is most commonly defined as:

$$
\mathrm{PRD}=\sqrt{\frac{\sum_{\mathrm{n}=1}^{\mathrm{N}}(\mathrm{x}(\mathrm{n})-\tilde{x}(\mathrm{n}))^{2}}{\sum_{\mathrm{n}=1^{\mathrm{N}}}^{\mathrm{N}} \mathrm{x}^{2}(\mathrm{n})}} \times 100
$$

Where $\mathrm{x}(\mathrm{n})$ and $\square \mathrm{x}(\mathrm{n})$ are the values of the original and reconstructed samples, respectively, and $\mathrm{N}$ is the length of the window over which the PRD is calculated.

\subsection{Compression Measurement}

The size of compression is often measured by $\mathrm{CR}$, which is defined as the ratio between the bit rate of the original signal $\left(b_{\text {original }}\right)$ and the bit rate of the compressed one $\left(b_{\text {compressed }}\right)$.

$$
\mathrm{CR}=\frac{\mathrm{b}_{\text {original }}}{\mathrm{b}_{\text {compressed }}}
$$

The problem of using the above definition of CR is that every algorithm is fed with an ECG signal that has a different sampling frequency and a different number of quantization levels; thus, the bit rate of the original signal is not standard. Some attempts were made in the past to define standards for sampling frequency and quantization, but these standards were not implemented, and developers of the algorithms still use rates and quantizers that are convenient to them. The number of bits transmitted per sample of the compressed signal has been used as a measure of information rate. This measure removes the dependency on the quantizer's resolution, but the dependence on the sampling frequency remains. Another way is to use the number of bits transmitted per second as a compression measure. This measure removes the dependence on the quantizer's resolution as well as the dependence on the sampling frequency.

\section{Result and Discussion}

In this section the performance of proposed compression algorithm are evaluated and compared with other known algorithms. For this purpose, data from the MIT-BIH arrhythmia database is used to evaluate the 
proposed compression algorithm. The database consists of normal sinus rhythm and different arrhythmia such as ectopic beats and different QRS complex morphologies. The distortion between the original and reconstructed signal is measured by percentage rms difference (PRD or PRD1). Although PRD does not exactly correspond to the result of a clinical subjective test, it is easy to calculate and compare, so it is widely used in the ECG data compression literature.

Fig. 1 and Fig. 2 are introduced to allow visual assessment of the quality of 1800 samples length ECG signal extracted from records 117 and 119 respectively.

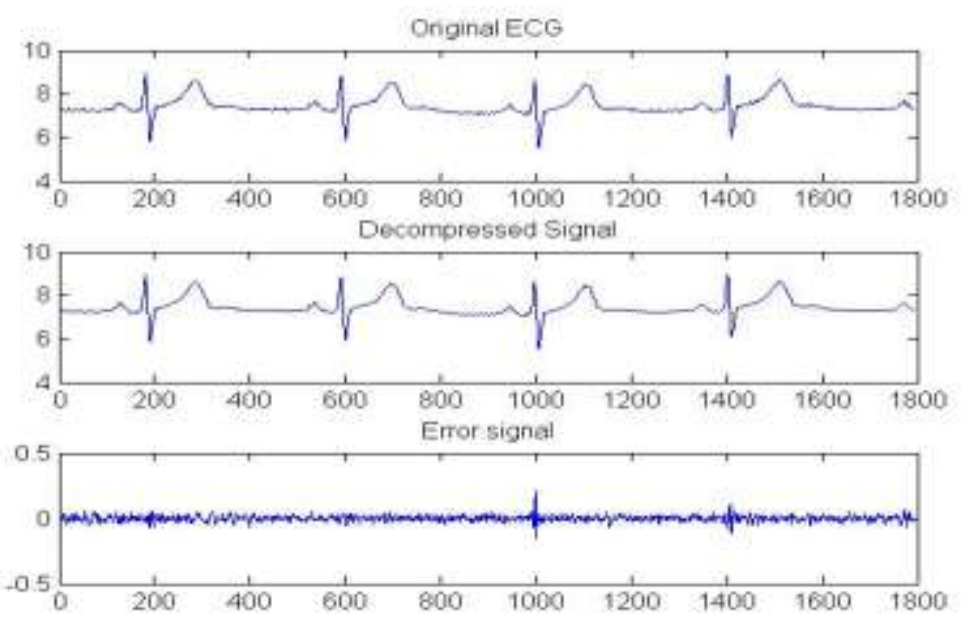

Figure1. Visual assessment of the quality of four second ECG signal from record 117.

(a) Original signal; (b) reconstructed signal; (c) error signal (with PRD=0.292).

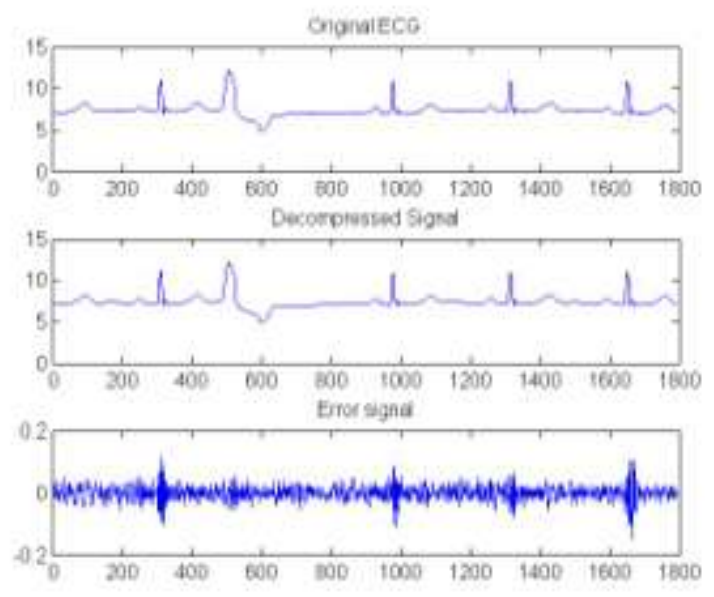

Figure 2.Visual assessment of the quality of four second ECG signal from record 119.

(a) Original signal; (b) reconstructed signal; (c) error signal (with PRD=0.307).

\section{Conclusion}

A hybrid scheme combining the spectral redundancy by compressing the subordinate components more than the dominant components then the resulting transformed coefficients that represent the transformational signal are then thresholded and compressed using a new coding technique for storage space saving. Improvement in performance parameters is achieved with the interaction of DCT with DWT transformation method, followed by thresholding and encoding.

The method here described has the advantage of better performance ratios. Besides the good performance in quality versus compression ratio, the proposed compression algorithm has some other features which are very important in the real-time environment. First, the reconstructed signal quality and the bit stream can be controlled by removing the spectral redundancy in subordinate components and/or the dominant components. Secondly, the computational complexity of the proposed codec is low, as witnessed by the recurrence relation of the DCT coefficients. 
Moreover, the proposed algorithm was also compared with some standards and already developed hybrid algorithms. It was observed that the proposed hybrid algorithm performs better than the existing algorithms.

\section{Acknowledgements}

We take this opportunity to thank our Head of Department Dr. A. D. Jadhav, and the entire Staff Members of E\&TC (PG) department for their co-operation and help during this work.

We are very much thankful to the Principal Dr. S. D. Lokhande for his support. Finally, I my honest and sincere feelings towards all those who directly or indirectly encouraged me, helped me, criticized me in accomplishment of my present work.

\section{References}

[1] http://www.physionet.org/physiobank/database/mitdb

[2] A Comparison of Single Lead ECG Data Compression Techniques.

[3] Al-Shrouf, A., Abo-Zahhad, M. and Ahmed, S.M., 2003, A novel compression algorithm for electrocardiogram signals based on the linear prediction of the wavelet coefficients. Digital Signal Processing, 13, 604-622.

[4] Sabah, M.A. and Abo-Zahhad, M., 2001, A new hybrid algorithm for ECG signal compression based on the wavelet transformation. Medical Engineering and Physics, 24, 50-66

[5] Abo-Zahhad, M. and Rajoub, B.A., 2002, an effective coding technique for the compression of one-dimensional signals using wavelets. Medical Engineering and Physics, 24, 185-199.

[6] Manikandan, M.S. and Dandapat, S., 2007, Wavelet thresholding based ECG compression using USZZQ and Huffman coding of DSM. Biomedical Signal Processing and Coding, 1, 261-270.

[7] Miaou, S. and Yen, H., 2000, Quality driven gold washing adaptive vector quantization and its application to ECG data compression. IEEE Transactions on Biomedical discrete cosine transform and discrete wavelet transform for ECG signal compression has been presented \& their combination removes Engineering, 47, 209-218.

[8] Leonardo Vidal Batista a, b, c, *, Elmar Uwe Kurt Melcher a, Luis Carlos Carvalho b, 2001, Compression of ECG signals by optimized quantization of discrete cosine transform coefficients, Medical Engineering \& Physics 23 (2001) 127-134

[9] Zigel, Y., Cohen, A. and Kats, A., 2000, the weighted diagnostic distortion (WDD) measure for ECG signal compression. IEEE Transactions on Biomedical Engineering, 47, 1422-1430.

[10] Lu, Z., Kim, D. and Pearlman, W., 2000, Wavelet compression of ECG signals by set partitioning in hierarchical trees algorithm. IEEE Transactions on Biomedical Engineering, 47, 849-856.

[11] Hilton, M., 1997, Wavelet and wavelet packet compression of electrocardiograms. IEEE Transactions on Biomedical Engineering, 44, 394-402.

[12] Y. Chompusri_and S. Yimman, 2009, Energy Packing Efficiency Based Threshold Level Selection for DTW ECG Compression, International Journal of Applied Biomedical Engineering vol.2, no.2 2009.

[13] http://ieeeexplore.org/

[14] www.sciencedirect.com 